\title{
Efecto del entrenamiento de músculos abdominales sobre la fuerza muscular respiratoria y flujos espiratorios forzados en adolescentes sanos sedentarios
}

a. Laboratorio de Biología del Ejercicio (LBE), Escuela de Kinesiología, Facultad de Ciencias de la Salud, Universidad San Sebastián, Concepción, Chile.

b. Programa de Magíster en Kinesiología Cardiorrespiratoria,

Escuela de

Kinesiología, Facultad de Ciencias de la

Salud, Universidad

San Sebastián,

Concepción, Chile.

c. Escuela de

Kinesiología, Facultad

de Salud, Universidad

Santo Tomás,

Concepción, Chile.

d.Grupo de

Investigación en Salud

Cardiovascular y

Respiratoria IDEAS-

CVR, Concepción,

Chile.

e. Programa de

Doctorado en Ciencias

Médicas, Facultad de

Medicina, Universidad

de La Frontera,

Temuco, Chile.

f. Departamento de Cirugía y Centro de Excelencia en Estudios Morfológicos y

Quirúrgicos (CEMyQ),

Universidad de La

Frontera, Chile.

g. Centro de

Investigación

en Biociencias,

Universidad

Autónoma de Chile, Chile.

Correspondencia:

Klgo. Iván Rodríguez

Núñez,

ivan.rodriguez@uss.cl

Financiamiento:

Este estudio fue financiado por la Escuela de Kinesiología de la Universidad San Sebastián, Concepción, Chile.

Conflicto de intereses: Ninguno que declarar.

Recibido: 15-11-2015

Aceptado: 20-4-2016

\section{Effect of abdominal muscle training on respiratory muscle strength and forced expiratory flows in sedentary, healthy adolescents}

\author{
Klgo. Iván Rodríguez-Núnezz $z^{a, b, c, d, e}$, Klga. Ximena Navarro ${ }^{b}, K_{l}$ lgo. Darwin Gatica ${ }^{c, d, e}, y$ \\ Dr. Carlos Manterola $a^{e, f g}$
}

\section{RESUMEN}

Introducción. El entrenamiento muscular respiratorio es el método más utilizado para revertir la debilidad muscular respiratoria; no obstante, el efecto de protocolos basados en maniobras no respiratorias no ha sido suficientemente estudiado en población pediátrica. El objetivo de este estudio fue determinar el efecto del entrenamiento de la musculatura abdominal sobre la fuerza de los músculos respiratorios y los flujos espiratorios forzados en adolescentes sanos.

Métodos. Estudiocuasiexperimental. La muestra estuvo integrada por adolescentes sanos divididos en dos grupos: un grupo experimental, quienes realizaron 8 semanas de entrenamiento de la musculatura abdominal activo, y un grupo control equivalente. Se midió la fuerza de los músculos abdominales, la presión inspiratoria máxima, la presión espiratoria máxima (PeMax), el flujo espiratorio máximo y el flujo máximo de tos antes y después del protocolo. Se consideró significativo un valor de $\mathrm{p}<0,05$.

Resultados. Todas las variables estudiadas aumentaron significativamente en el grupo experimental; sin embargo, solo la PeMax se incrementó en el grupo control. Adicionalmente, el grupo experimental presentó una mayor PeMaxqueel grupo control al final del protocolo, así como también una mayor ganancia en la PeMax y flujo espiratorio máximo. Finalmente, el incremento en la PeMax se asoció con el aumento en el flujo máximo de tos en el grupo experimental, lo que no fue observado en el grupo control.

Conclusión. En 8 semanas de entrenamiento de la musculatura abdominal, se logró incrementar la PeMax y el flujo espiratorio máximo en adolescentes sanos (sedentarios). Estos efectos se asociaron a incrementos en el flujo máximo de tos inducidos por la intervención.

Palabras clave: entrenamiento del músculo respiratorio, flujo espiratorio máximo, músculos respiratorios, músculos abdominales, tos.

http:/ /dx.doi.org/10.5546/aap.2016.434

\section{INTRODUCCIÓN}

La debilidad de los músculos respiratorios es una de las principales repercusiones funcionales de los niños con enfermedades respiratorias crónicas (ERC) ${ }^{1-3}$ Esto conduce al desarrollo de hipoventilación alveolar, formación de microatelectasias y disfunción del mecanismo de la tos, factores que aumentan el riesgo de falla respiratoria. ${ }^{4}$

Se ha observado que el entrenamiento de los músculos respiratorios (EMR) sería una estrategia efectiva para mitigar el deterioro de su fuerza y resistencia, ${ }^{5-7}$ así como también el flujo máximo de tos (FMT) en niños con enfermedad neuromuscular. ${ }^{6}$ Pese a ello, aún no existe consenso respecto a los protocolos de entrenamiento más efectivos para el logro de los objetivos funcionales. ${ }^{8}$ En el contexto clínico, el EMR mediante la utilización de una válvula umbral (Threshold ${ }^{\circledR}$ ) corresponde al método más ampliamente utilizado; ${ }^{7}$ sin embargo, esta modalidad constituye un método indirecto e inespecífico de fortalecimiento muscular.

Desde el punto de vista fisiológico, el trabajo de los músculos respiratorios se encuentra influenciado por la acción tanto de musculatura torácica como abdominal. De esta forma, se ha demostrado que diversas modalidades de ejercicios localizados en la musculatura abdominal, los miembros superiores y los miembros inferiores estimulan al diafragma, 
incrementan la presión transdiafragmática e inducen diversos grados de fatiga de músculos abdominales ${ }^{9,10}$

Estos antecedentes subrayan el potencial efecto de protocolos de entrenamiento basados en ejercicios no respiratorios sobre la fuerza de este grupo muscular y parámetros de función respiratoria. Así, diversos estudios realizados en adultos han demostrado que maniobras no respiratorias, como flexiones anteriores de tronco, pueden aumentar la fuerza de los músculos respiratorios. ${ }^{11}$ Adicionalmente, se ha observado que protocolos de entrenamiento pasivo (basados en estimulación eléctrica) $)^{12,13}$ y activo de los músculos abdominales serían efectivos para mejorar la fuerza de los músculos respiratorios y flujos espiratorios forzados. ${ }^{14} \mathrm{Sin}$ embargo, el efecto de este tipo de estrategias de entrenamiento no ha sido suficientemente estudiado en población pediátrica. ${ }^{14}$

Por lo tanto, el objetivo de este estudio fue evaluar el efecto del entrenamiento activo de la musculatura abdominal sobre la fuerza de los músculos respiratorios y los flujos espiratorios forzados en adolescentes sanos sedentarios.

\section{MATERIAL Y MÉTODO}

El presente estudio posee un diseño cuasi experimental con un grupo control equivalente. Los resultados preliminares del grupo experimental fueron reportados en un estudio piloto previamente publicado. ${ }^{14}$

Muestra: A través de un muestreo por conveniencia, se seleccionaron adolescentes sanos, sin restricción de sexo, estudiantes de un colegio público de la ciudad de Concepción, Chile. Como criterios de inclusión, fueron considerados adolescentes sanos sedentarios (que realizaran actividad física con una frecuencia menor de dos veces por semana por 60 minutos) y que no hubieran sido sometidos a ejercicios de fortalecimiento de algún grupo muscular en el último mes. Como criterios de exclusión, se consideraron la existencia de alguna patología cardiorrespiratoria o musculoesquelética y tabaquismo. Los criterios de elegibilidad fueron verificados mediante la entrevista a cada uno de los participantes del estudio, así como también una lista de chequeo, que fue completada directamente por sus padres antes de ingresar al estudio. No se realizó ningún otro control cardiovascular, como ecocardiograma o ergometría, antes del protocolo experimental. Tanto los participantes del estudio como sus padres firmaron asentimiento y consentimiento informado, respectivamente, y el estudio fue autorizado por el Comité de Ética Institucional. Se realizó durante los meses de junio de 2014 y mayo de 2015.

La estimación del tamaño de la muestra fue realizada de acuerdo con la metodología publicada previamente. ${ }^{14}$ Se consideró una potencia estadística de $80 \%$ y un riesgo de error tipo 1 de 5\%; de este modo, la muestra mínima necesaria para conducir este estudio fue de 17 sujetos por grupo. En este contexto, una vez seleccionado el grupo experimental de acuerdo con los criterios de inclusión, se seleccionó el grupo control. Ambos grupos fueron emparejados por características biodemográficas (peso, estatura, edad y género) y variables de función respiratoria (fuerza muscular respiratoria y flujos espiratorios forzados) antes del protocolo.

Variables: Antes de iniciar el estudio, fueron registrados la edad, el sexo, la estatura y el peso corporal. Para la medición del peso y la estatura, se utilizó una cinta métrica y una balanza análoga, cuyo registro se expresó en kilogramos $(\mathrm{kg})$ y centímetros $(\mathrm{cm})$, respectivamente.

Como variables de interés, se consideraron la fuerza de los músculos respiratorios y los flujos forzados del sistema respiratorio. La fuerza de los músculos respiratorios se determinó a través de la presión inspiratoria máxima (PiMax), medida a través de un esfuerzo inspiratorio máximo mantenido por, al menos, un segundo desde el volumen residual, y la presión espiratoria máxima (PeMax), medida a través de un esfuerzo espiratorio máximo mantenido por, al menos, un segundo desde la capacidad pulmonar total. Para estas mediciones, se utilizó un vacuómetro aneroide marca NS120-TRS calibrado en centímetros de agua (desde 0 a -120 y desde 0 a $+120 \mathrm{cmH}_{2} \mathrm{O}$ ). Los valores obtenidos de PiMax y PeMax se expresaron en valores absolutos $\left(\mathrm{cmH}_{2} \mathrm{O}\right){ }^{15}$

Por otra parte, tanto el flujo espiratorio máximo (FEM) como el flujo máximo de tos (FMT) fueron medidos con un flujómetro (MiniWright ${ }^{\circledR}$; Clement Clarke International, Essex, England). El FEM se llevó a cabo con el sujeto en posición de pie, utilizando una pinza nasal, que debía soplar con la mayor fuerza posible desde la capacidad pulmonar total. La prueba se realizó un mínimo de tres ocasiones y un máximo de ocho; se registró el valor más alto reproducible en tres intentos con una diferencia no mayor del 10\% entre cada uno de ellos. ${ }^{16}$ El FMT se midió con el 
sujeto sentado; se le indicó que inspirara lo más profundo posible y que realizara un esfuerzo de tos máximo a través del flujómetro. Los resultados se expresaron en litros/minutos. ${ }^{17}$

Adicionalmente, como una medida de efectividad del EMA, fue determinada la fuerza de los músculos abdominales (FMA). Esta se midió mediante el Sit-Up Test, el cual corresponde a una prueba funcional, ampliamente utilizada tanto en sujetos sanos como en pacientes con ERC. ${ }^{14,18}$ En la posición inicial, el sujeto se halla tendido en decúbito supino, las rodillas en flexión de 90 grados con la planta de los pies tocando el suelo y las manos a un costado de la cabeza. Un ayudante mantiene los pies fijos en la colchoneta. En este momento, el examinador indica el comienzo de la prueba, que consiste en efectuar el número máximo de ciclos de flexión-extensión de tronco durante 30 segundos.

Protocolo de entrenamiento: El entrenamiento de músculos abdominales (EMA) se llevó a cabo de acuerdo con lo publicado por Rodríguez y cols. ${ }^{14}$ Brevemente, el protocolo consistió en 8 semanas de entrenamiento con ejercicios específicos para cada grupo muscular de la faja abdominal. En cada sesión de ejercicio, fueron trabajados todos los grupos musculares de manera activa, sin asistencia externa, y se alcanzó un rango de 200 a 300 repeticiones por sesión. Se realizaron dos sesiones por semana y cada una tuvo una duración de 45 minutos. Por su parte, a los integrantes del grupo control se les solicitó la realización de sus actividades normales y fueron monitoreados durante las 8 semanas de protocolo experimental. Finalmente, tanto las variables de función respiratoria como la FMA fueron medidas un día antes y un día después del protocolo de entrenamiento, en ambos grupos estudiados.

Plan de análisis: En el software estadístico MedCalc Statistical Software versión 14.12.0 (MedCalc Software bvba, Ostend, Belgium; http:/ / www.medcalc.org, 2014), se realizó un análisis exploratorio de los datos y un cálculo de normalidad mediante el test de ShapiroWilk. Una vez verificada la existencia de la distribución normal de los datos, se realizó una estadística descriptiva mediante el cálculo de promedio y error estándar de la media (EEM).

Además, para comparar el valor absoluto de los parámetros de función respiratoria entre los grupos de estudio, fue utilizado el t-test para muestras independientes, así como también el t-test para muestras pareadas, para evaluar las diferencias entre los valores absolutos antes y después del protocolo de intervención.

TABLA 1. Características basales de la muestra

\begin{tabular}{lccc}
\hline Variables & Control $(\mathbf{n}=\mathbf{2 3})$ & Experimental $(\mathbf{n}=\mathbf{1 7})$ & Valor $\boldsymbol{p}$ \\
\hline Sexo $(\mathrm{H} / \mathrm{M})$ & $9 / 14$ & $8 / 9$ & 0,8588 \\
Edad $(\mathrm{años})$ & $15,4 \pm 0,1$ & $15,2 \pm 0,1$ & 0,1609 \\
Estatura $(\mathrm{cm})$ & $161,4 \pm 1,9$ & $162,9 \pm 1,7$ & 0,5601 \\
Peso $(\mathrm{kg})$ & $61,6 \pm 2,3$ & $56,6 \pm 2,4$ & 0,1537 \\
\hline
\end{tabular}

H: hombre; M: mujer. Los resultados se muestran en promedio \pm error estándar de la media.

TABLA 2. Fuerza muscular respiratoria y flujos respiratorios forzados antes y después del entrenamiento

\begin{tabular}{lccc}
\hline Variables & Control $(\mathbf{n}=\mathbf{2 3})$ & Experimental $(\mathbf{n}=\mathbf{1 7})$ & Valor $\boldsymbol{p}$ \\
\hline Pre-PiMax $\left(\mathrm{cmH}_{2} \mathrm{O}\right)$ & $99,6 \pm 5,9$ & $96,5 \pm 4,3$ & 0,6828 \\
Pos-PiMax $\left(\mathrm{cmH}_{2} \mathrm{O}\right)$ & $108,1 \pm 4,1$ & $112,8 \pm 4,3^{* *}$ & 0,4051 \\
Pre-PeMax $\left(\mathrm{cmH}_{2} \mathrm{O}\right)$ & $84,5 \pm 3,9$ & $69,4 \pm 3,8$ & 0,0799 \\
Pos-PeMax (cm $\left.\mathrm{cm}_{2} \mathrm{O}\right)$ & $93,5 \pm 2,0^{* * *}$ & $104,3 \pm 3,3^{* * *}$ & 0,0125 \\
Pre-FEM (L/min) & $416,5 \pm 19,9$ & $424,3 \pm 22,6$ & 0,4379 \\
Pos-FEM (L/min) & $424,5 \pm 15,8$ & $457,0 \pm 19,0^{* *}$ & 0,0759 \\
Pre-FMT (L/min) & $382,2 \pm 18,5$ & $401,2 \pm 19,0$ & 0,4898 \\
Pos-FMT (L/min) & $410,3 \pm 13,7$ & $437,6 \pm 19,2^{*}$ & 0,2520 \\
Pre-Sit-up test (n. ${ }^{\circ}$ rep./30 s) & $23,3 \pm 1,4$ & $23,5 \pm 1,0$ & 0,4592 \\
Post-Sit-up test (n. ${ }^{\circ}$ rep./30 s) & $23,4 \pm 1,7$ & $28,6 \pm 0,8^{* * *}$ & 0,0039 \\
\hline
\end{tabular}

PiMax: presión inspiratoria máxima; PeMax: presión espiratoria máxima; FEM: flujo espiratorio máximo; FMT: flujo máximo de tos; Sit-up test: cantidad de repeticiones efectuadas en 30 segundos. Los resultados se muestran en media \pm error estándar de la media. ${ }^{*}<0,05 ;{ }^{* *}<0,01 ;{ }^{* * *}<0,001$. 
Adicionalmente, fue utilizado el test U de MannWhitney para comparar la magnitud de las variaciones en los valores de las variables de interés entre el grupo control y experimental. La asociación entre variables dicotómicas fue evaluada mediante el test de chi-cuadrado.

Finalmente, se realizó un análisis de regresión lineal con el fin de determinar la asociación entre la magnitud del efecto sobre la PeMax y los flujos espiratorios forzados (FEM y FMT).

Se consideró significativo un nivel de $\mathrm{p}<0,05$.

\section{RESULTADOS}

Características generales de la muestra: $\mathrm{Al}$ estudio ingresaron 17 sujetos ( 8 varones) en el grupo experimental y 23 sujetos (9 varones) en el grupo control. La edad, el sexo y las características antropométricas se detallan en la Tabla 1, y se evidencia una similitud de los grupos en estudio.

Además, no existió una diferencia significativa en cuanto al nivel de fuerza muscular respiratoria (PiMax, PeMax), flujos espiratorios forzados (FEM y FMT) y FMA entre los grupos antes de iniciar el protocolo de entrenamiento (Tabla 2).
Efecto del entrenamiento: Todos los sujetos realizaron el protocolo y lo finalizaron sin incidentes. No hubo exclusiones durante el protocolo por falta de adherencia o problemas de salud de alguno de los integrantes de ambos grupos. Luego del programa de entrenamiento, solo los sujetos del grupo experimental incrementaron en $21,7 \%$ la cantidad de abdominales ejecutados en 30 segundos $(p=0,0001)$.

En el grupo experimental, tanto la fuerza muscular respiratoria como los flujos espiratorios forzados aumentaron significativamente respecto al valor basal. Así, la PiMax se incrementó en $16,8 \%$ ( $\mathrm{p}=0,006)$; la PeMax, en 50,2\% ( $<<0,0001)$. El FEM aumentó en $8,4 \%(p=0,003)$ y el FMT, en $9,1 \%(p=0,015)$. Por su parte, en el grupo control, estas variables se incrementaron levemente; el aumento fue significativo solo en la variable PeMax (+16\%; $\mathrm{p}=0,0004)$.

En el análisis entre los grupos, luego de la intervención, se observó un mayor nivel de fuerza muscular respiratoria y flujos espiratorios forzados en el grupo experimental; no obstante,

FIgURA 1. Magnitud del efecto inducido por el entrenamiento de músculos abdominales comparado con el grupo control
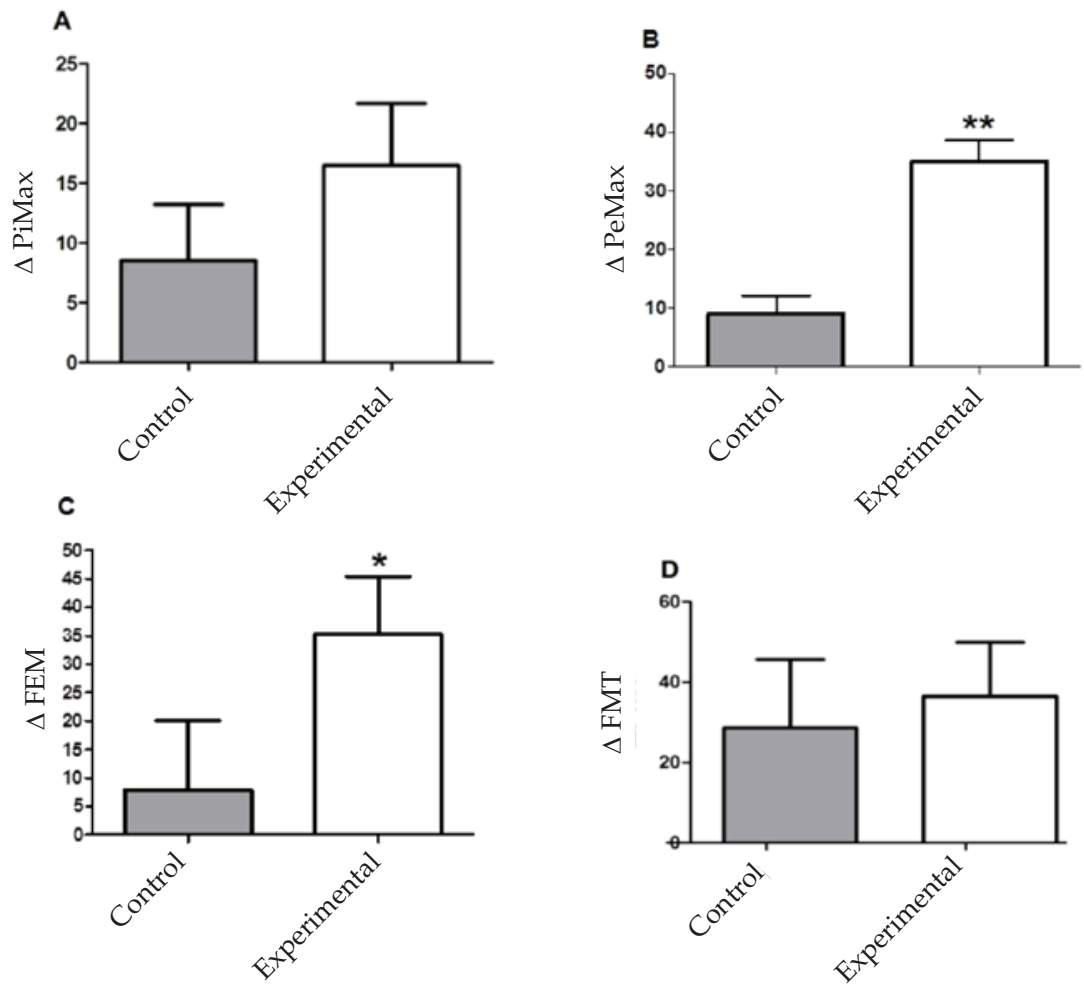

En A, se muestra el efecto sobre la presión inspiratoria máxima (PiMax); en B, sobre la presión espiratoria máxima (PeMax); en $\mathrm{C}$, sobre el flujo espiratorio máximo (FEM); y, en D, sobre el flujo máximo de tos (FMT). ${ }^{*} p<0,05$; ${ }^{* *} p<0,0001$. 
solo en la variable PeMax, esta diferencia fue estadísticamente significativa $(+14,2 \% ; \mathrm{p}=0,0125)$.

Además, al analizar la magnitud del efecto en las variables estudiadas, se observó, en el grupo experimental, una mayor ganancia funcional en todas las variables, comparado con el grupo control; la ganancia fue estadísticamente significativa solo en la PeMax ( $<<0,0001)$ y FEM $(\mathrm{p}=0,02)$ (Figura 1).

En relación con el análisis de regresión lineal entre las ganancias en la PeMax y los flujos espiratorios forzados (FEM y FMT), se observó una correlación positiva entre la PeMax y el FMT $\left(r=0,66 ; r^{2}=0,44 ; p=0,0038\right)$ en el grupo experimental. Esto no fue observado en el grupo control (pendiente grupo experimental: 2,4; IC 95\%: 0,9-3,9; pendiente grupo control: 0,7; IC 95\%: de -1,7 a 3,18) (Tabla 3 - Figura 2). No se observó asociación entre las ganancias de la PeMax y FEM $(\mathrm{p}=0,12)$.

\section{DISCUSIÓN}

Los principales hallazgos de este estudio revelaron que el EMA fue efectivo para incrementar la fuerza de los músculos respiratorios, principalmente, la fuerza muscular espiratoria (PeMax) y el FEM. A su vez, las ganancias en la PeMax inducidas por el entrenamiento se asociaron con las ganancias en la función de tos en el grupo experimental.

Estos resultados concuerdan con aquellos que han evaluado el efecto de maniobras no respiratorias sobre el rendimiento de los músculos respiratorios. Strongoli y cols., demostraron que maniobras, como la bipedestación y flexión de tronco desde posición sedente y supina, incluyen la musculatura respiratoria e incrementan significativamente la presión diafragmática a un nivel suficiente para inducir un estímulo de entrenamiento. ${ }^{9}$ DePalo y cols. demostraron

TABLA 3. Análisis de regresión lineal entre la magnitud del efecto sobre la presión espiratoria máxima y el flujo máximo de tos

\begin{tabular}{lccccc}
\hline Grupo & Pendiente $^{*}$ & Intercepto & $\mathbf{r}$ & $\mathbf{r}^{2}$ & Valor $p$ \\
\hline Experimental & $2,5($ de 0,9 a 3,9) & $-49,5($ de $-107,3$ a 8,4) & 0,66 (de 0,27 a 0,87) & 0,43 & 0,0038 \\
Control & 0,72 (de -1,7 a 3,2) & 22,1 (de -20,3 a 64,4) & $0,13($ de -0,29 a 0,51) & 0,02 & 0,5486 \\
\hline
\end{tabular}

Los resultados son expresados en valor absoluto y $95 \%$ de intervalo de confianza.

FIGURA 2. Regresión lineal entre la magnitud del efecto sobre el flujo máximo de tos y la presión espiratoria máxima

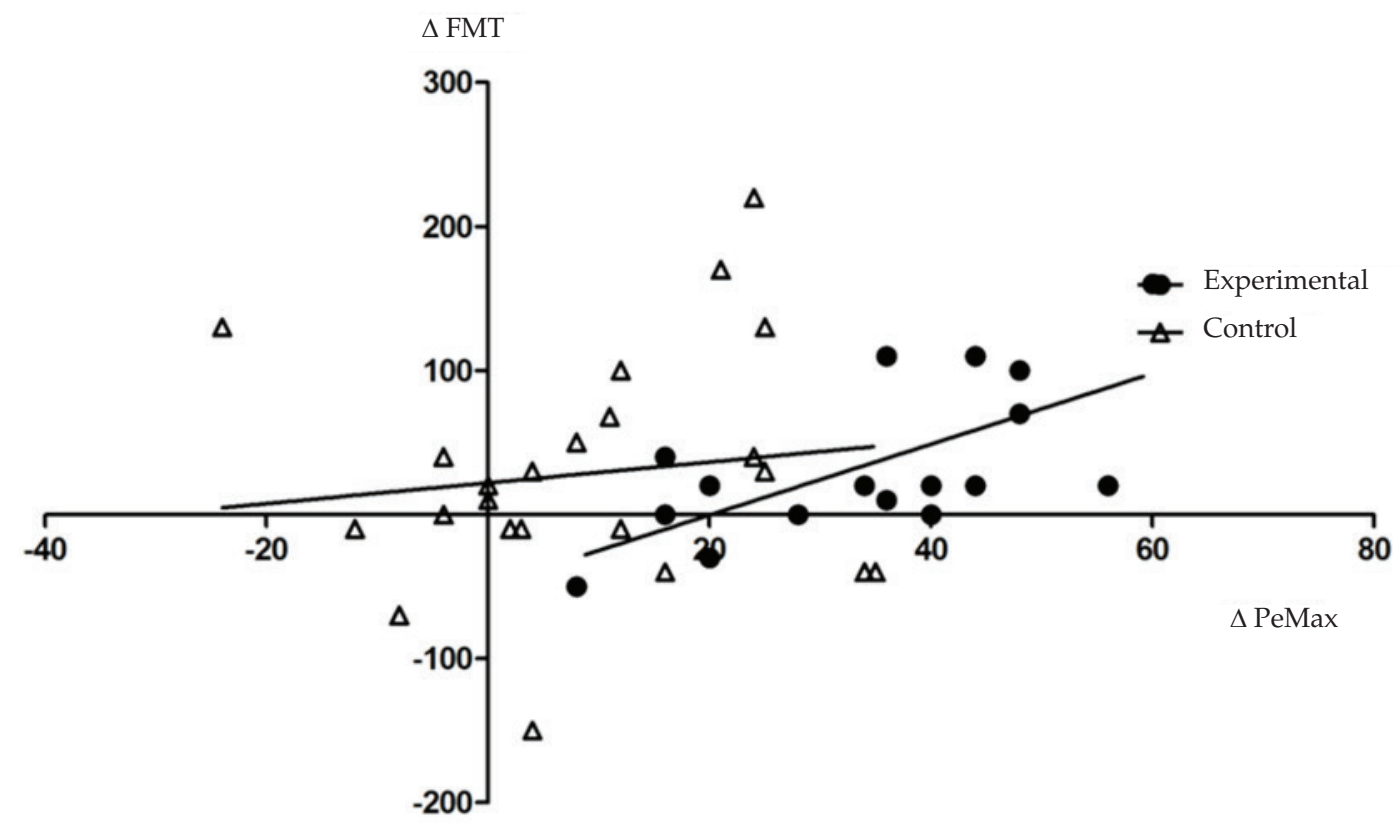

FMT: flujo máximo de tos; PeMax: presión espiratoria máxima.

* Variable eje Y: ganancia en el flujo máximo de tos inducida por entrenamiento;

variable eje X: ganancia en la presión espiratoria máxima inducida por entrenamiento. 
que 16 semanas de entrenamiento de flexión de bíceps y tronco se asociaron a incrementos significativos de la PiMax $(+27,6 \%)$ y PeMax $(+36,9 \%) .{ }^{11}$ En nuestro estudio, se puso en evidencia que la ejecución de ejercicios específicos de fortalecimiento abdominal favorecería, principalmente, los grupos musculares implicados en la espiración activa; no obstante, los resultados no fueron concluyentes para los efectos sobre los músculos inspiratorios.

Además, resultados similares han sido reportados con protocolos convencionales de EMR, los cuales han mostrado ser efectivos para mejorar la fuerza de los músculos respiratorios y el rendimiento físico en sujetos sanos desentrenados y atletas. ${ }^{19,20}$ En el contexto clínico, se ha reportado que el entrenamiento de los músculos inspiratorios mejora tanto la fuerza como la resistencia muscular respiratoria en niños con enfermedad neuromuscular,; $\mathrm{e}$, incluso, protocolos de entrenamiento domiciliario (no supervisados) inducen incrementos en la fuerza de los músculos implicados en la respiración, con efectos positivos sobre los flujos espiratorios forzados. ${ }^{6}$

Adicionalmente, el EMA incrementó significativamente el FEM y el FMT en el grupo experimental; no obstante, la magnitud del efecto fue significativamente superior al grupo control sólo en el FEM. Pese a lo anterior, fue observada una asociación significativa entre las ganancias en la PeMax y FMT. En este ámbito, ha sido observado previamente que 6 semanas de estimulación eléctrica de músculos abdominales junto con maniobras de tos voluntaria serían efectivas para incrementar el FMT y las presiones abdominales y gástricas durante la espiración forzada. ${ }^{13}$ Por su parte, Gollee et al., observaron que la estimulación funcional superficial de los músculos abdominales incrementaba el FMT y el volumen corriente de sujetos con tetraplejia. ${ }^{21}$ En el análisis de regresión lineal realizado en nuestro estudio, fue calculado por cada $\mathrm{CmH}_{2} \mathrm{O}$ de incremento en la PeMax un aumento del FMT en 2,4 L/min, lo que confirma la estrecha interdependencia entre ambas variables. En términos biomecánicos, durante el mecanismo de la tos, los músculos espiratorios cumplen un rol crucial, tanto al relajarse durante la fase inspiratoria como al contraerse de manera isométrica durante la fase compresiva, lo que permite incrementar la presión intraabdominal hasta $300 \mathrm{mmHg}{ }^{22}$
Entre las limitaciones de este estudio, podemos comentar que el establecimiento de un grupo control equivalente asignado de manera arbitraria, sin asignación aleatoria, podría constituir una potencial fuente de sesgo. En este contexto, se intentó emparejar de acuerdo con parámetros relevantes (variables antropométricas y función respiratoria); sin embargo, no podemos descartar la existencia de alguna otra covariable, no controlada, que pueda afectar la homogeneidad de los grupos.

En relación con la confiabilidad de las variables de interés, en el grupo control, fueron observadas ciertas diferencias en los valores obtenidos antes y después del protocolo de intervención, principalmente, en la variable PeMax. En este sentido, estudios previos han mostrado distintos niveles de confiabilidad en la medición de variables de función respiratoria $\mathrm{y}$, específicamente, moderada concordancia test retest en la PeMax (diferencia test retest de $\left.10 \mathrm{cmH}_{2} \mathrm{O}\right) .{ }^{17}$ Moran y cols., observaron una diferencia de $10 \mathrm{cmH}_{2} \mathrm{O}$ en la PeMax $(p<0,05),{ }^{23}$ lo cual concuerda con lo observado en nuestro estudio. No obstante ello, en el grupo experimental, existió una mayor ganancia funcional en la variable PeMax respecto al grupo control. Esto permite establecer que el incremento en la fuerza de los músculos espiratorios en el grupo experimental se debió al efecto inducido por el protocolo de entrenamiento desarrollado.

Por su parte, la PiMax se incrementó de manera significativa luego de la intervención; sin embargo, la diferencia entre la magnitud de los cambios inducidos por el EMA comparado con el grupo control no fue significativa. Por lo tanto, no es posible descartar la potencial influencia del azar en la magnitud de las diferencias observadas entre grupos.

\section{CONCLUSIONES}

Finalmente, es posible concluir que 8 semanas de EMA fueron suficientes para incrementar el FEM y la fuerza muscular respiratoria, principalmente, la PeMax, en adolescentes sanos sedentarios. Estos efectos son asociados a los cambios en el FMT inducidos por la intervención.

Además, la PiMax aumentó significativamente luego del EMA en el grupo experimental; no obstante, la magnitud del efecto no mostró diferencias con el grupo control. Estudios ulteriores deben ser efectuados para confirmar si el EMA produce algún efecto en la fuerza muscular inspiratoria. 


\section{Agradecimientos}

A cada uno de los adolescentes y sus padres por aceptar participar del estudio, así como también al establecimiento educacional por permitirnos desarrollar este proyecto.

A los estudiantes de Kinesiología que han colaborado en esta línea de investigación.

\section{REFERENCIAS}

1. Neve V, Cuisset JM, Edme JL, Carpentier A, et al. Sniff nasal inspiratory pressure in the longitudinal assessment of young Duchenne muscular dystrophy children. Eur Respir J 2013;42(3):671-80.

2. Dassios TG, Katelari A, Doudounakis S, Dimitriou G. Chronic Pseudomonas aeruginosa infection and respiratory muscle impairment in cystic fibrosis. Respir Care 2014;59(3):363-70.

3. Rodríguez I, Arriagada R, Fuentes C, Zenteno D. Aspectos fisiopatológicos de la rehabilitación respiratoria en fibrosis quística. Neumol Pediatr 2012;7(2):51-7.

4. Racca F, Del Sorbo L, Mongini T, Vianello A, et al. Respiratory management of acute respiratory failure in neuromuscular diseases. Minerva Anestesiol 2010;76(1):5162 .

5. Koessler W, Wanke T, Winkler G, Nader A, et al. 2 Years' experience with inspiratory muscle training in patients with neuromuscular disorders. Chest 2001;120(3):765-9.

6. RodríguezI,ZentenoD,Manterola C. Effects of home-based respiratory muscle training in children and adolescents with chronic lung disease. J Bras Pneumol 2014;40(6):62633.

7. Rodríguez I, Fuentes C, Rivas C, Molina F, et al. Rehabilitación respiratoria en el paciente neuromuscular: efectos sobre la tolerancia al ejercicio y musculatura respiratoria. Resultado de una serie de casos. Rev Chil Enferm Respir 2013;29(4):196-203.

8. Zenteno D, Puppo H, Vera R, Torres R, et al. Guías de rehabilitación para niños con enfermedades respiratorias crónicas. Neumol Pediatr 2007;3(Supl 1):25-33.

9. Strongoli LM, Gomez CL, Coast JR. The effect of core exercises on transdiaphragmatic pressure. J Sports Sci Med 2010;9(2):270-4.

10. Suzuki J, Tanaka R, Yan S, Chen R, et al. Assessment of abdominal muscle contractility, strength, and fatigue. Am J Respir Crit Care Med 1999;159(4 Pt 1):1052-60.
11. DePalo VA, Parker AL, Al-Bilbeisi F, McCool FD. Respiratory muscle strength training with nonrespiratory maneuvers. J Appl Physiol (1985) 2004;96(2):731-4.

12. Lee BB, Boswell-Ruys C, Butler JE, Gandevia SC. Surface functional electrical stimulation of the abdominal muscles to enhance cough and assist tracheostomy decannulation after high-level spinal cord injury. J Spinal Cord Med 2008;31(1):78-82.

13. McBain RA, Boswell-Ruys CL, Lee BB, Gandevia SC, et al. Abdominal muscle training can enhance cough after spinal cord injury. Neurorehabil Neural Repair 2013;27(9):834-43.

14. Rodríguez I, Alarcón M, Gutierrez C, Hermosilla P, et al. Efecto del entrenamiento de músculos abdominales sobrela función respiratoria en adolescentes sanos: Estudio piloto. Rev Chil Enferm Respir 2014;30(4):203-11.

15. American Thoracic Society/European Respiratory Society. ATS/ERS Statement on respiratory muscle testing. Am J Respir Crit Care Med 2002;166(4):518-624.

16. Céspedes J, Gutiérrez M, Oyarzún M. Flujometría en la práctica de atención primaria. Rev Chil Enferm Respir 2010;26(1):47-8.

17. Rodríguez I. Confiabilidad de la fuerza muscular respiratoria y flujos espiratorios forzados en adolescentes sanos. Rev Chil Enferm Respir 2015;31(2):86-93.

18. Elbasan B, Tunali N, Duzgun I, Ozcelik U. Effects of chest physiotherapy and aerobic exercise training on physical fitness in young children with cystic fibrosis. Ital J Pediatr 2012;38:2.

19. Illi SK, Held U, Frank I, Spengler CM. Effect of respiratory muscle training on exercise performance in healthy individuals: a systematic review and meta-analysis. Sports Med 2012;42(8):707-24.

20. HajGhanbari B, Yamabayashi C, Buna TR, Coelho JD, et al. Effects of respiratory muscle training on performance in athletes: a systematic review with meta-analyses. JStrength Cond Res 2013;27(6):1643-63.

21. Gollee H, Hunt KJ, Allan DB, Fraser MH, et al. Automatic electrical stimulation of abdominal wall muscles increases tidal volume and cough peak flow in tetraplegia. Technol Health Care 2008;16(4):273-81.

22. McCool FD. Global physiology and pathophysiology of cough: ACCP evidence-based clinical practice guidelines. Chest 2006;129(1 Suppl):48s-53s.

23. Moran F, Piper A, Elborn JS, Bradley JM. Respiratory muscle pressures in non-CF bronchiectasis: repeatability and reliability. Chron Respir Dis 2010;7(3):165-71. 